\title{
Comment 1.2
}

\section{LIEN VERBAUWHEDE KOGLIN}

Globally, there is a growing interest in the role of universities and public research institutes in the alchemy of innovation, the emphasis being on how they can make more systematic efforts to unlock the commercial value of their research. While many of these feel it is imperative that their knowledge transfer activities work to recover costs, from my experience, revenue generation, in most cases, is not and in my view should not be the primary motivation. The reasons these institutes engage in knowledge transfer is to advance education and research; and at the same time it helps to ensure that public investment in research is impactful, that it contributes to broader socioeconomic development objectives. However, the going is tough, even in high-income countries and the entrepreneurial character of these institutes remains the subject of academic scrutiny. The chapter inspires a deeper understanding of this critical area by examining the evolving role of institutes in national innovation systems. It also examines the impact of legislative and policy initiatives that promote protection of inventions through patenting and their commercialization through licensing and startup formation.

WIPO has developed several programs in an endeavor to help public research organizations set the right institutional policies in order to successfully harness public research for innovation and contribute to socioeconomic development in their regions. ${ }^{1}$ In this context, I have witnessed two particular trends concerning universities' engagement with IP-based commercialization, where improved understanding and additional metrics would seem to be desirable: (1) an expansion of academic incentive schemes and (2) an increased commitment to socially responsible commercialization.

${ }^{1}$ See WIPO's web page on universities and IP at https://www.wipo.int/about-ip/en/universities_research/. To support its activities, WIPO has created an IP Policy Template for Universities and Research Institutions, Guidelines for Customization of the IP Policy Template, and an IP Policy Drafters' Checklist. 


\section{Trend toward Actively Motivating and Empowering Researchers to Participate in Knowledge Transfer}

The direct involvement of academic researchers has proved to be a determinant in the success of knowledge transfer. This clearly calls for cultivating a culture that supports and encourages both invention disclosures and the participation of inventors in the transfer process. There is also a need for a better understanding of the strategies of the various types of inventor/researcher involved and their motivations to participate in the process.

To boost academic entrepreneurial activity, universities, and public research institutes are introducing an ever wider range of incentives for researchers, where IP and commercialization efforts receive greater rewards comparable to publications. Among the specific incentives are: generous royalty and equity terms; tying IP generation and research commercialization to career development; sufficient time to engage in IPrelated activities (leave of absence, course reductions, relief of admin responsibilities, etc.); research funding and infrastructure; internal commercialization support and mentoring; entrepreneurship education programs; recognition through awards and public acknowledgement, etc. Empirical findings seem to suggest that the influence of such incentives (both monetary and nonmonetary) is not always predictable, given the differences in motives, perspectives and cultures of the academic scientists.

Questions that merit further empirical investigation include:

- What drives academics to be engaged in the commercialization of their research outcomes?

- Which factors can have an impact on the attractiveness of academic incentives (such as differences between the researchers in terms of gender, age, research field, characteristics of the ecosystem in which they operate, the seniority of researchers)? Is it therefore possible that a variety of incentives may be required for different types of researchers?

- What is the effect of the royalty share allocated to researchers? A large share can potentially enhance technology licensing, whereas a lower share is more likely to boost spinoffs; at the same time, too low a share allocated to the institution may not be sufficient to cover overall costs and may challenge the quality of services their knowledge transfer offices (KTO) provide.

- Is giving inventors a share of the equity in a spinoff rather than a simple share of returns a more effective way to motivate, considering 
the higher levels of uncertainty over returns, but also the prospect of higher returns than might accrue to licensing?

- What kind of remuneration packages are necessary to attract highly skilled employees at the KTO, noting that internal policies may prevent institutes from providing competitive salaries?

- How can the efficiency of institutes' support services be improved (e.g., by creation of an association of KTOs to pool support efforts)?

- How does the existence of competing incentives affect the engendering of an entrepreneurship culture, considering that researchers tend to have multiple "principals" (mainly the university itself, heads of departments, KTO, research council, government and external agencies) who often incentivize different outputs?

To evaluate the effectiveness of their incentives program, institutes must also establish comprehensive and systematic performance indicators, including some specific to IP-based commercialization. Empirically grounded metrics are critically important to an effective incentive structure. An important caveat is that incentive structures tend to be too focused on the supply side, which is the ability of the university to transfer knowledge. Attention needs also to be paid to the demand side, which involves the demand from industry for assistance in resolving problems and the region's ability to absorb the research results.

\section{Trend toward Socially Responsible Research Commercialization}

It would appear that entrepreneurial institutions around the world face more pressure to be responsive to the needs of society and environmental issues. The growing concern of this social dimension of higher education calls for resolute efforts to devise strategies that will establish them as drivers of societal well-being, while identifying the right indicators to monitor socioeconomic benefit flowing from such engagement. Successful cases prove that institutes have the means at their disposal to integrate a social dimension in their knowledge transfer practices (including those that are IPR-based), such as creating research programs directed to solving social and environmental problems; anticipating which technologies may have applications that address important unmet social needs; adopting socially responsible licensing provisions that increase the availability of medicines and environmental technologies in developing countries; retaining the right to grant additional licenses to manufacturers of generic drugs; negotiating licensing terms 
that allow third parties to access and distribute the innovation and its derivative products; promoting the creation of spinoffs; participation in community-based research; etc. In addition to those, the chapter presents a set of levers for preventing the potentially negative impacts of IPRbased knowledge transfer.

While policymakers and institutes tend to collect and employ mostly quantitative performance indicators to capture scientific productivity and commercial outcomes, the local/regional impact of universities and public research institutes extends far beyond knowledge transfer and tangible outputs (in terms of human capital attraction, formation of entrepreneurship capital within a locale, informal networks, new ideas, etc.). However, as the chapter indicates, establishing clear causal relationships between IPR-based knowledge transfer and these societal benefits is hard. Accordingly, statistics on the number of licenses issued or the number of spinoffs established do not effectively do justice to answering the question of how institutions address tangible socioeconomic outcomes.

Despite the fact that there is a trend afoot in some high-income countries to assess the success of knowledge transfer using alternative criteria, such as social impact or contribution to welfare, there is still no consensus on a set of systematic social impact measurements.

\section{Concluding Observations}

There is no magic formula for harnessing public research for innovation, given that different factors and levels of support interventions affect knowledge transfer outcomes. At the same time, there are magical "elements" or "factors" that the success stories have in common. The chapter does a nice job in elucidating such success factors at the country and institutional level. It is, however, important to note that success is a result of more collaborative efforts within an innovation ecosystem. For example, Yale's success in creating the biotech cluster is to a large extent due to the fact that it implemented changes in collaboration with other players in the region, to push for local economic impact. Countries also need to put into practice initiatives that promote and strengthen academia and business collaborations. One example in Brazil is the ITec platform, which was financed by the Ministry of Science \& Technology and counts on the participation of companies and universities to feed the framework of demands and offers. 
At the micro/institutional level, two efforts deserve closer attention and empirical investigation, namely, getting appropriate incentive structures and the commitment to socially responsible commercialization.

The transformation of institutes to become more entrepreneurial may be supported by creating new incentives and performance-linked criteria for researchers. How academic incentives work, and how they can be used to achieve intended results, remains a contested issue. In the university and public research institute contexts, the pursuit of science and innovation driven by external incentives, especially financial rewards, is considered by some as going against the traditional values of academia. However, international experience shows that institutionalizing an efficient incentive program is a critical precondition for increasing opportunities for commercializing university inventions. The challenge for institutes lies in selecting the types of incentive and their associated metrics, based on the institute's mission, culture, and goals, and the country's innovation ecosystem.

Socially responsible entrepreneurship is in large part a cultural attribute. Institutes can do their bit to encourage its development by, for instance, formulating policies that promote ethically acceptable and socially desirable knowledge transfer coupled with appropriate performance indicators. It would appear that institutes still struggle with (1) defining what "socially responsible" means and (2) measuring the extent to which their socially responsible policies and practices have meaningful impact. Maintaining a system of comprehensive indicators, including variables that can also measure social impact, is crucial for any country, regardless of its level of development, to help institutes better evaluate their roles in the creation of regional innovation and social value through research commercialization. 